\title{
The experimental study of energy dissipation during fatigue crack propagation under biaxial loading
}

\author{
Oleg Plekhov, Aleksei Vshivkov, Anastasia Iziumova \\ Institute of Continuous Media Mechanics Russian Academy of Sciences Ural Branch, Russia \\ poa@icmm.ru,vshivkov.a@icmm.ru,fedorova@icmm.ru
}

Aleksandr Zakharov, Valery Shlyannikov

Kazan Scientific Center of Russian Academy of Sciences, Russia

alex.2akharov88@mail.ru,shlyannikov@mail.ru

\begin{abstract}
The work is devoted to experimental study of heat flux evolution at the fatigue crack tip during biaxial loading with a goal to relate the heat flux to the rate of crack propagation under different loading conditions. The plane samples of titanium alloy (Grade 2) $1 \mathrm{~mm}$ thick were weakened by notch to initiate fatigue crack at their centers. Infrared thermography and the contact heat flux sensor, which is based on the Seebeck effect, were used to monitor the dissipated thermal energy. The samples were subject to cyclic loading with constant stress amplitude at different biaxial coefficients. The experimental results confirmed the previous conclusions of the authors about two regime of energy dissipation at fatigue crack tip under Paris regime. At the first stage, the power of heat flux is proportional to the product of the crack rate by the crack length. The second stage is characterized by a traditional linear relationship between the crack rate and the heat flux.
\end{abstract}

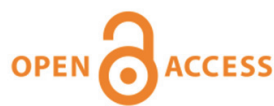

Citation: Plekhov, O., Vshivkov, A., Iziumova, A., Zakharov, A., Shlyannikov, V., The experimental study of energy dissipation during fatigue crack propagation under biaxial loading, Frattura ed Integrità Strutturale, 48 (2019) 50-57.

Received: 28.11 .2018

Accepted: 30.01 .2019

Published: 01.04.2019

Copyright: (C) 2019 This is an open access article under the terms of the CC-BY 4.0, which permits unrestricted use, distribution, and reproduction in any medium, provided the original author and source are credited.

KEYwORDS. Fatigue; Biaxial loading; Crack propagation; Dissipated energy.

\section{INTRODUCTION}

I $\mathrm{n}$ the age of rapid technical progress, highly sophisticated mechanisms and technologies and ambitious projects in the field of mechanical engineering, aircraft construction, nuclear energy and space exploration much effort has been put into intensive development of many fields of science and technologies, including fracture mechanics. A number of approaches has been developed to study the processes of nucleation and propagation of fatigue cracks in metals [1-4]. It is well known that real metals have a complex structure, which is a hierarchy of different scale levels. Under deformation, the structural changes are observed at all scale levels and leads to irreversible deformation and failure, which is accompanied by accumulation and dissipation of energy. The investigation into thermodynamics of deformation and failure is a key issue of solid mechanics. The analysis of the kinetics of damage accumulation, the process of crack nucleation and kinetics of the crack growth allows specialists to predict the time of structure failure and to perform in 
proper time a partial replacement or repair of fractured structural elements. Moreover, the repair or replacement of the defective parts on a timely basis is more effective than their complete replacement after mechanical damage. It is therefore very important to know the time during which the flaw size in the ill-behaved areas reach critical values.

The actual engineering structures operate under complex types of loading. So, it is of considerable interest to study the behavior of materials under mixed loading conditions that combine mode 1 and mode 2 fractures. In sufficiently plastic structural materials the propagation of the crack begins when the plastic deformation near its tip becomes large (of the order of 10 percent). This irreversible process is accompanied by the release and accumulation of energy, which leads to a local temperature change in the region of the crack tip and the occurrence of a heat flux.

For a long time, infrared thermography is regarded as the most effective technique for estimating the power of the heat sources in the process of mechanical testing [5,6]. A principal solution to the problem of measuring the energy dissipated in the structure under deformation and failure can be obtained by the development of additional system for direct monitoring of the heat flux [7]. The heat generation process depends on both the thermo elastic effect and plastic energy dissipation. The measurement of heat flux near the crack tip allows one to calculate the energy balance during crack propagation and to develop a new equation for its description. Attempts to develop a new equation for crack propagation were made by many authors. They used such quantities as the J-integral, the work of plastic deformation, the size of the zone of plastic deformation, the amount of dissipated energy and others [8-11]. The classical assumption of almost complete dissipation of the deformation energy into heat [12] has proved to be correct only in a limited number of cases. The previous authors' investigations were focused on the problem of crack growth under the opening or mode I fracture mechanism (see [13]). In this work, a relationship for the growth rate of a fatigue crack was developed based on the analysis of the energy balance at its tip. However, failure of most structures occurs under the mixed mode loading. Many materials, structures and components subject to uniaxial loads often contain randomly oriented defects and cracks, which lead to a mixed mode state due to rotation about the loading axis.

This study is devoted to the investigation of the energy dissipation in the process of crack propagation under biaxial loading. For this purpose, an original contact heat flux sensor was developed to record energy dissipation during crack propagation under biaxial loading and to verify the data of infrared thermography. This sensor made it possible to study in details the dissipated energy evolution in metal samples (titanium alloy Grade 2) in bi-axial loading tests and to determine the relationships between the energy dissipation and the fatigue crack rate.

\section{EXPERIMENTAL SETUP}

series of samples made of titanium alloy Grade 2 (chemical composition is presented in Tab. 1) were tested in $A$ the servo-hydraulic biaxial test system Biss BI-00-502 at the Kazan Scientific Center of the Russian Academy of 1 Sciences. The photo of experimental setup is given in Fig. 2. The geometry of the samples is shown in Fig. 1. During tests the samples were subject to cyclic loading of $10 \mathrm{~Hz}$ at constant stress amplitude and for different biaxial coefficient $\eta=\mathrm{Px} / \mathrm{Py}(1,0.7,0.5)$ and stress ratio $\mathrm{R}=\sigma_{\min } / \sigma_{\max }(0.1,0.3,0.5)$. Phases of biaxial loading coincided during the fatigue test. The experimental program is presented in Tab. 2. Crack length was measured by optic microscope.

\begin{tabular}{cccccccc}
\hline Fe & $\mathrm{C}$ & $\mathrm{Si}$ & $\mathrm{N}$ & $\mathrm{Ti}$ & $\mathrm{O}$ & $\mathrm{H}$ \\
0.25 & 0.07 & 0.1 & 0.04 & $99.24-99.7$ & 0.2 & 0.01 & \\
\hline
\end{tabular}

Table 1: The chemical composition of titanium alloy.

\begin{tabular}{ccccccc}
\hline Sample & $\mathrm{Sp} 1$ & $\mathrm{Sp} 2$ & $\mathrm{Sp} 3$ & $\mathrm{Sp} 4$ & $\mathrm{Sp} 5$ & $\mathrm{Sp} 6$ \\
Biaxial coefficient & 1 & 1 & 0.5 & 0 & 1 & 1 \\
Stress ratio & 0.1 & 0.1 & 0.1 & 0.1 & 0.5 & 0.5 \\
\hline
\end{tabular}

Table 2: Experimental program.

In the course of the experiment the crack length was measured by the optical microscopy method. To analyze the energy dissipation at the crack tip a contact heat flux sensor was designed and assembled. The sensor is based on the Seebeck effect, which is the reverse of the Peltier effect. 
The Peltier effect is a thermoelectric phenomenon, in which the passage of electric current through a conducting medium leads to the generation or absorption of heat at the point of contact (junction) of two dissimilar conductors. The quantity of heat and its sign depend on the type of contacting materials, the direction and the strength of the electric current. The quantity of heat absorbed or dissipated by the element is directly proportional to the current intensity and the time of current passage through the element:

$$
P=\Pi_{A B} I
$$

where $\mathrm{P}$ is the power of heat flux, $\mathrm{I}$ is the direct current, $\Pi_{A B}$ is the Peltier coefficient.

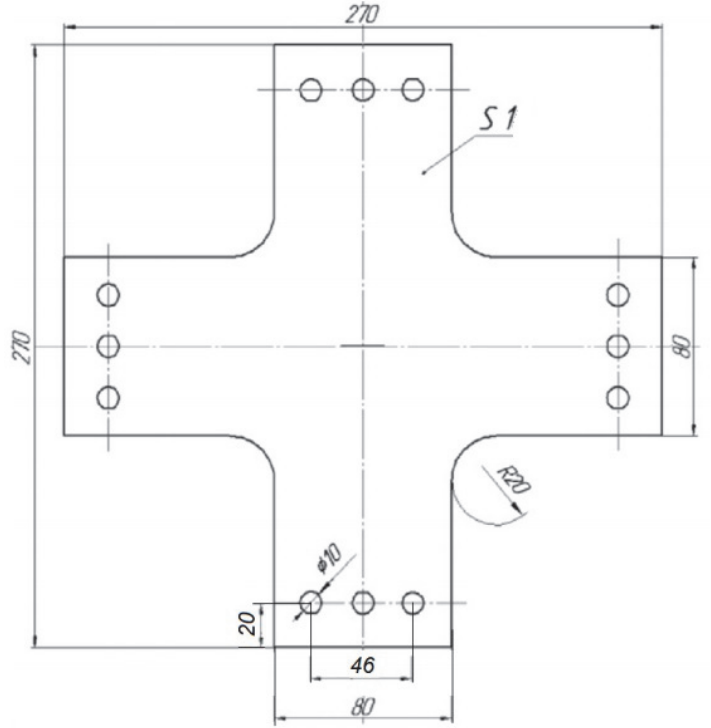

Figure 1: Geometry of samples, thickness is $1 \mathrm{~mm}$ (all Figure. 2. Testing machine Biss BI-00-502, Biaxial test sizes in millimeters).

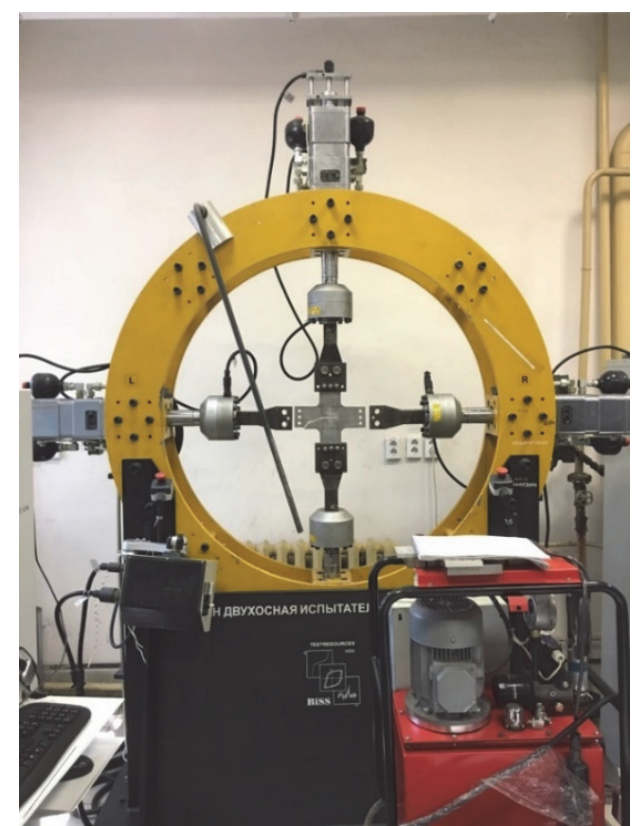

System.

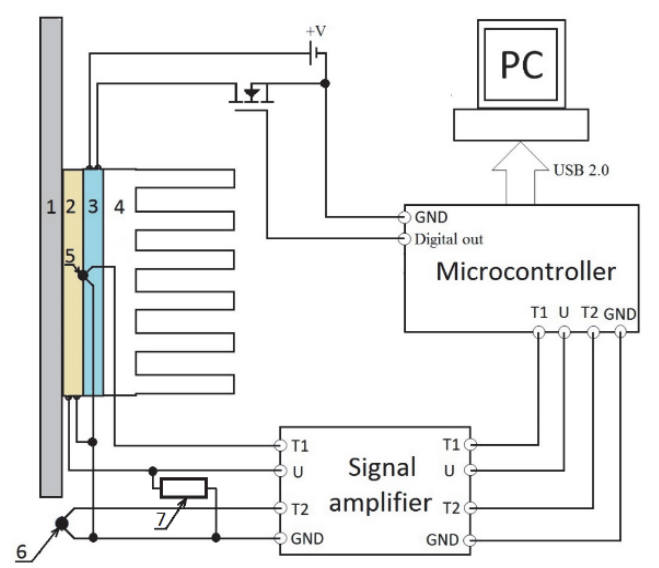

Figure 3: Schematic representation of the device.1 - testing sample; 2 - "measuring" Peltier element; 3 - "cooling" Peltier element; 4 - radiator; 5, 6- thermocouple; 7 - resistor.

Fig. 3 presents a schematic diagram of the heat flux sensor. Here, the following notation is used: 1 stands for a sample, 2 stands for a heat flux sensor. The application of a thermal paste provides good thermal contact between the sample and the sensor. Structurally, the sensor comprises two Peltier elements ("measuring"2 and "cooling"3), thermocouples 5, 6 and the radiator 4. The measuring Peltier element is connected to a low-resistance resistor 7 of 1.2 Om. To measure the heat 
flow through the "measuring" Peltier element during the experiment, the temperature on its free surface should be kept constant. The "cooling" Peltier element coupled with a radiator was connected to the "measuring" Peltier element. This cooling system has a feedback and is controlled with the two temperature sensors located between the "measuring" and "cooling" Peltier elements. It is placed at a certain distance from the examined sample in the zone with constant temperature.

The signal from the sensor (voltage at the resistor 7) is measured by the amplifier and registered by the ADC of the microcontroller. The data are transmitted from the microcontroller to the personal computer for further processing. The "cooling" Peltier element is controlled via pulse width modulation.

This sensor was calibrated using a device with a controlled heat flux. A wire resistor with the preset resistance was glued on a plastic plate, the size of which was equal to that of the test samples. The heat isolating system provided the heat flux only from the resistance to the sensor. The heat flow was calculated using the values of the resistor voltage and the electric current across the resistor.

The evolution of the temperature field was recorded by an infrared camera FLIR SC 5000. The spectral range of the camera was 3-5 $\mu \mathrm{m}$. The maximum frame size was $320 \times 256$ pixels; the spatial resolution was $10-4$ meters. The temperature sensitivity was $25 \mathrm{mK}$ at $300 \mathrm{~K}$. Calibration of the camera was made based on the standard calibration table. The FLIR SC5000 MW G1 F/3.0 close-up lens (distortion is less than $0.5 \%$ ) were used to investigate the plastic zone in details.

\section{RESULTS}

$\mathrm{D}$ uring experiments, a series of samples was subject to biaxial load tests with the aim to record the crack length and the heat flux. The crack propagation rate was $10^{-7}-10^{-4} \mathrm{~m} /$ cycle. A comparison of the methods used for estimating the heat flux from the crack tip is given in Figs. 4 and 5. The heat flux sensor made it possible to measure the integral heat flux and to verify the infrared thermography data. The infrared thermography method was used to obtain the temperature field and the field of heat source distribution in the crack tip region. Fig. 4 presents the characteristic curve describing time variation of the heat flux during the fatigue tests: solid line - the heat flux measured by the sensor, circles - the heat flux measured by the infrared thermography method with averaging over time and space occupied by the contact sensor. A comparison of heat fluxes measured by the two methods is given in Fig.5 (one point of infrared thermography from Fig. 4): green line - infrared thermography, red line - heat flux sensor. Figs. 4, 5 confirm the reliability of the heat flow measurement.

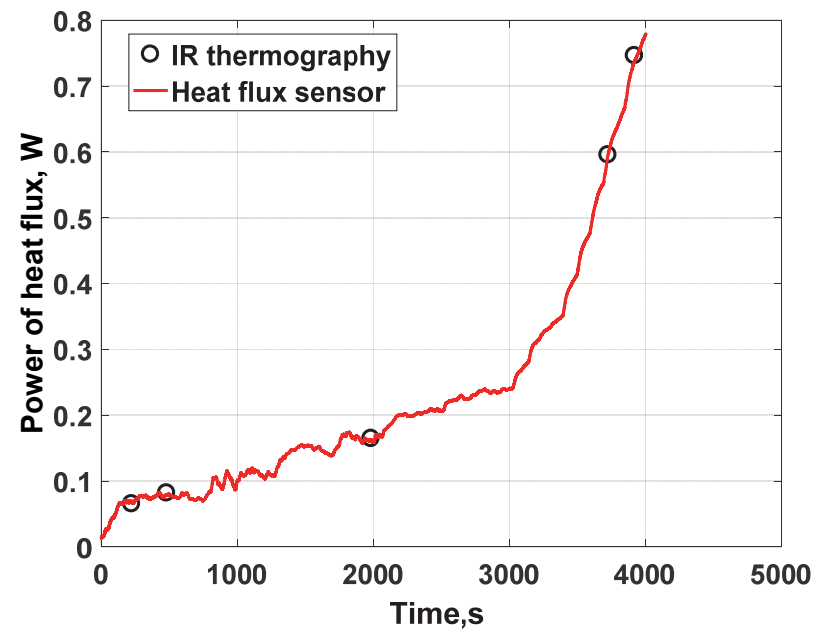

Figure. 4. Characteristic time dependence of heat flux.

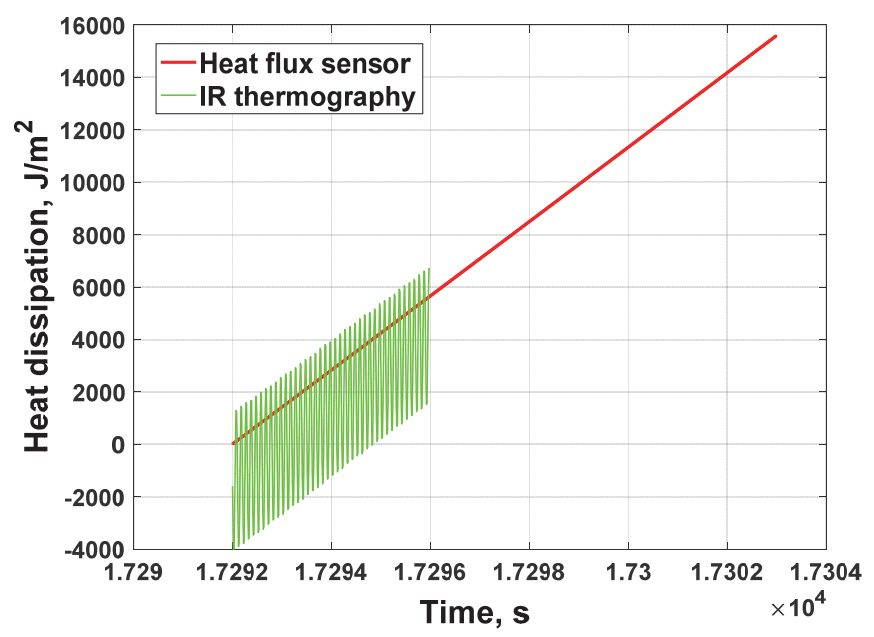

Figure. 5. Compare the infrared thermography and heat flux sensor.

In the previous work [13], the study of the fatigue crack propagation under uniaxial loading in the Paris regime revealed two stages of crack propagation differing in the character of energy dissipation. On the curve of the crack length and Paris curve, the point of change of the stages is clearly not pronounced. 
The time, at which the type of energy dissipation changes, is determined from the maximum value of the curvature of the function $\mathrm{q}(\mathrm{t})$ as:

$$
q(t)=\frac{\left|t^{\prime \prime}\right|}{\sqrt[3]{1+\left(t^{\prime}\right)^{2}}}
$$

where $\mathrm{q}(\mathrm{t})$ is the time dependence of the heat flux, $\mathrm{t}$ is time.

The crack length and crack rate are shown in Fig. 6. The heat flux curves illustrating two stages of crack propagation under biaxial loading are shown in Fig. 7. Circle markers show the point of changes of heat flux stages.

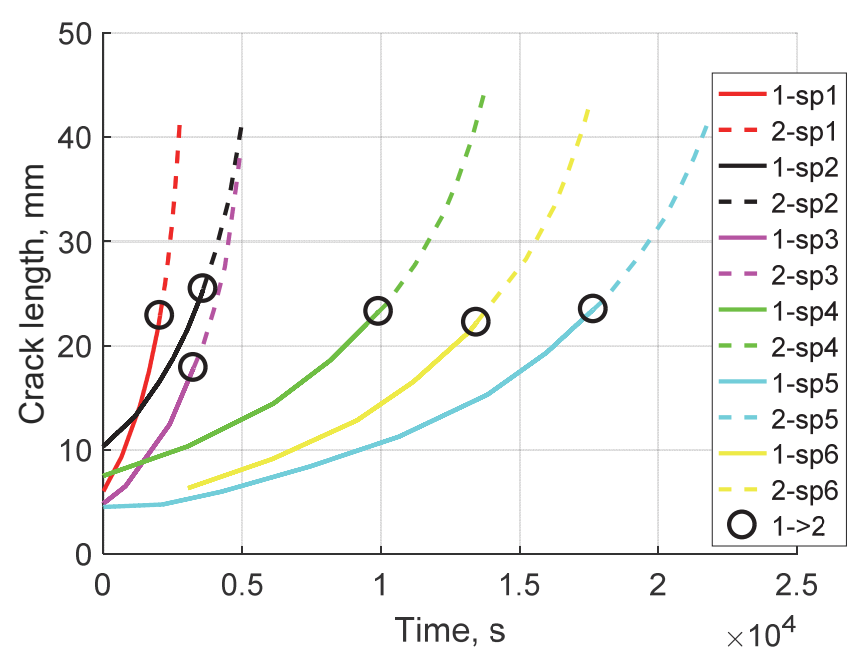

a)

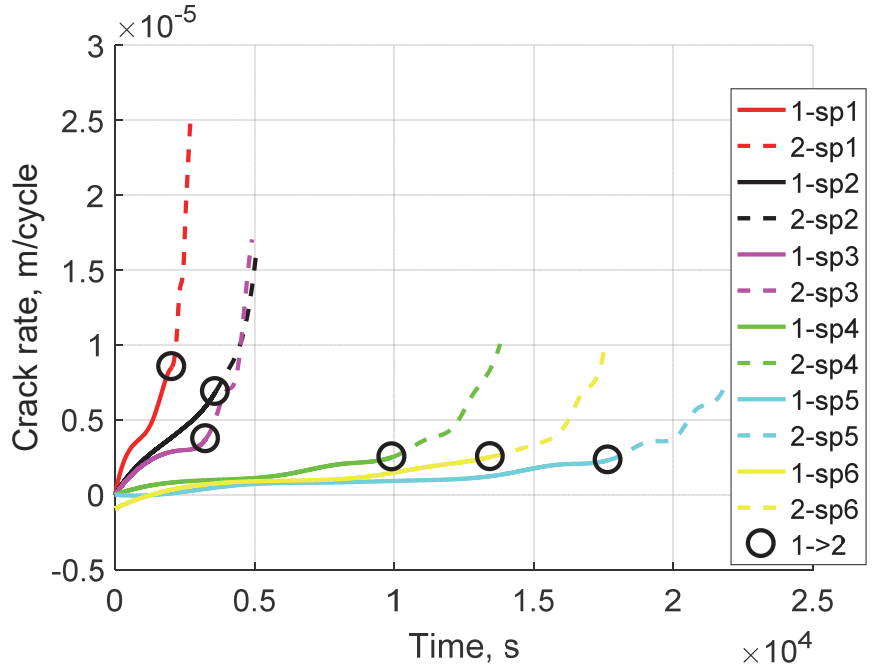

b)

Figure. 6. Crack length (a) and crack rate (b) during the fatigue test.

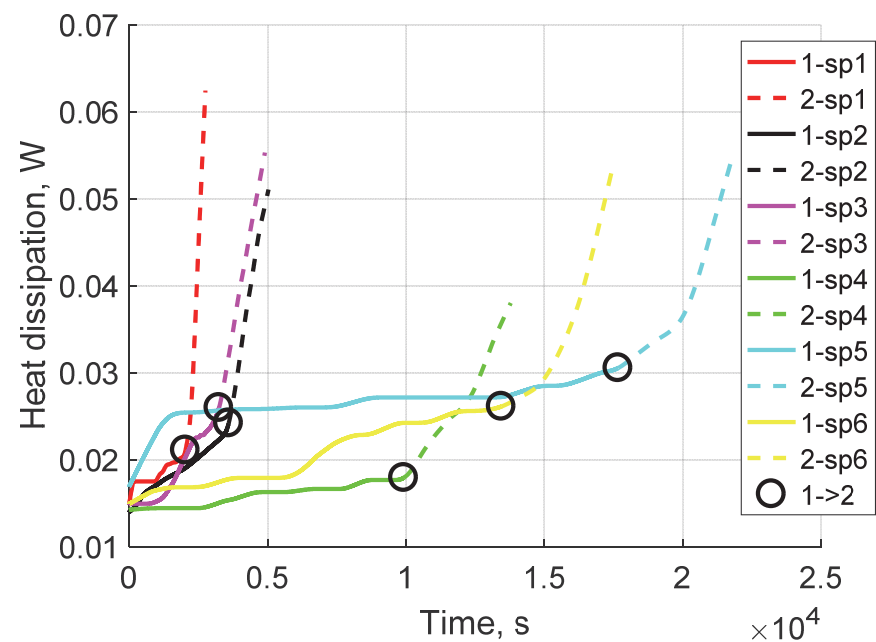

a)

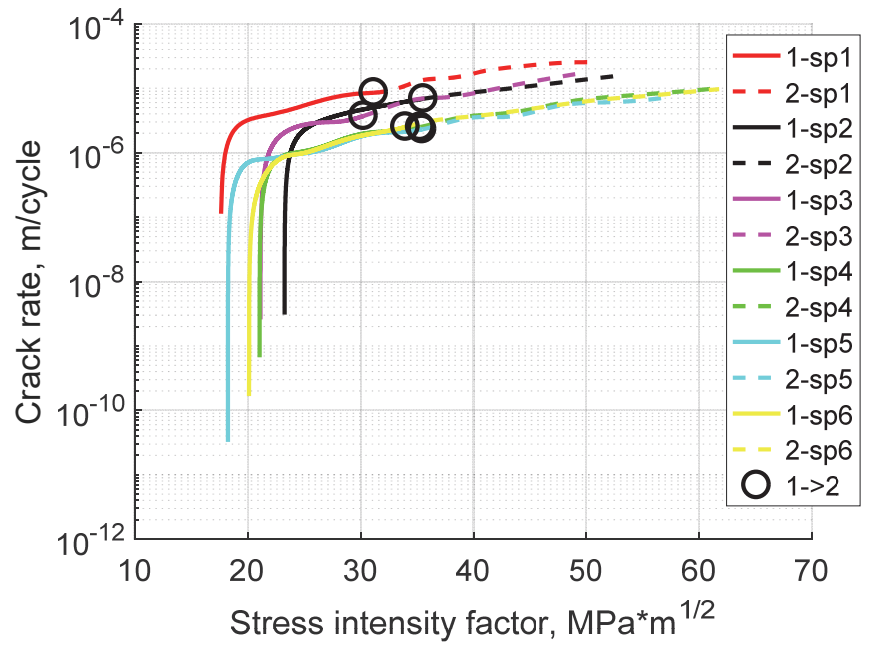

b)

Figure. 7. Two stages of fatigue crack propagation: energy dissipation (a), Paris curve (b).

The analysis of the experimental data in the light of the hypothesis about the stages of fatigue crack propagation [11] allowed us to derive characteristic relationships between the crack growth rate and the energy dissipation in both stages. 
At the first stage, the crack rate is proportional to the power of heat flux and the crack length $\left(\frac{d a}{d N} \sim Q_{1} a_{1}\right)$, and in the second stage - to the power of heat flux $\left(\frac{d a}{d N} \sim Q_{2}\right)$. The characteristic dependences are shown in Fig. 8.

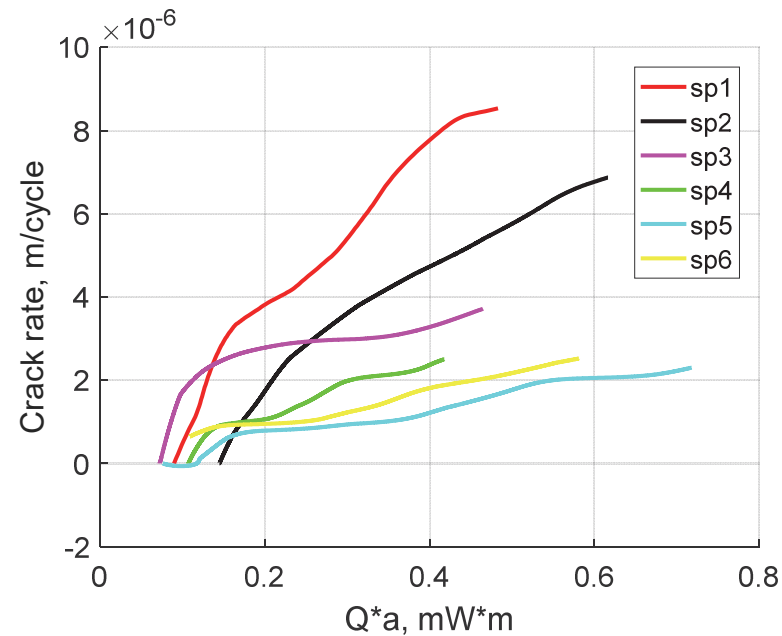

a)

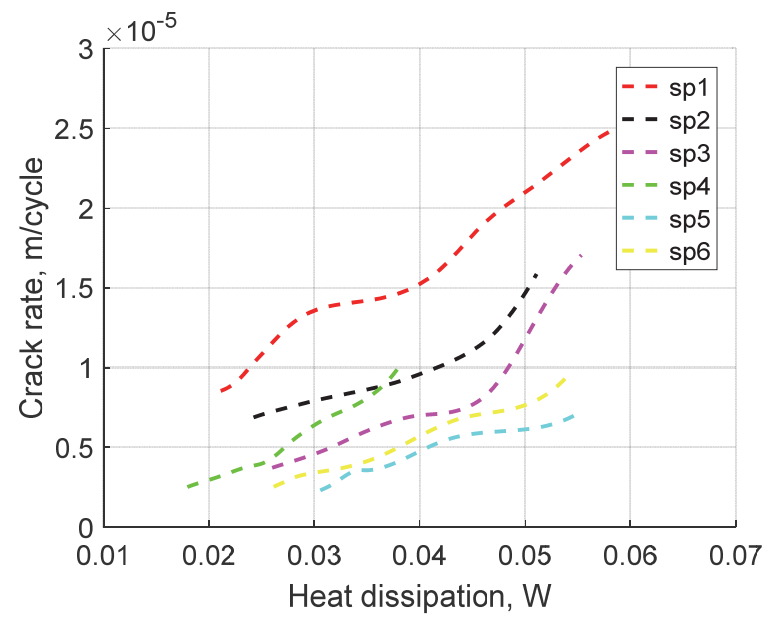

b)

Figure 8: Normalized heat flux and crack rate in the first (a) and second (b) stages.

It is worth noting that the relations used for the heat flux and the crack growth rate at both stages are consistent with the classical Paris regime. Fig. 9 describes the crack growth rate in terms of the stress intensity factor (SIF) and energy dissipation (the $\mathrm{K}$ index in the legend means the stress intensity factor, $\mathrm{Q}$ is the energy dissipation, 9a corresponds to the first stage, $9 \mathrm{~b}-$ to the second stage).

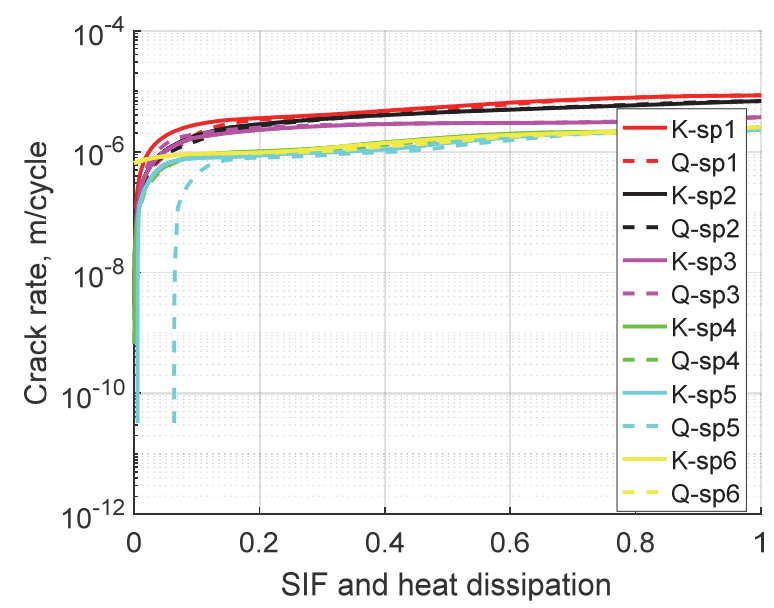

a)

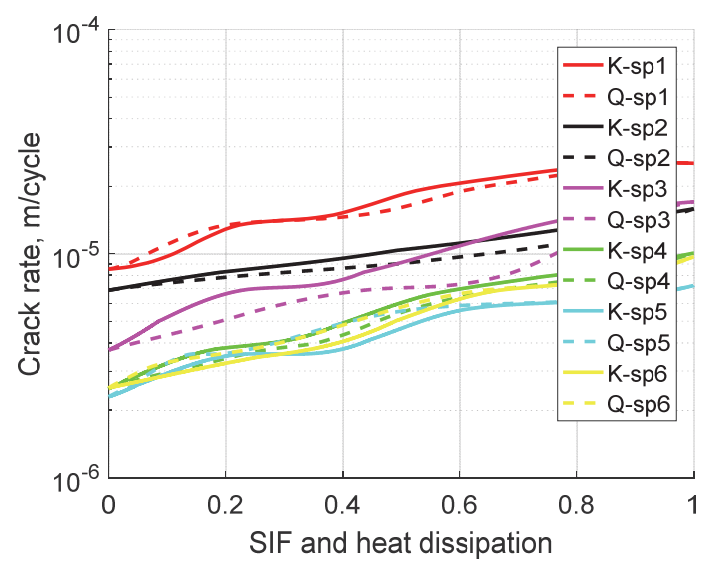

b)

Figure 9: Comparison between the energy approach and the classical assumption based on the stress intensity factor in the first (a) and second (b) stages.

In Fig. 9 normalization (3) for heat flux and normalization (4) for stress intensity factor are used:

$$
Q_{1} a_{1}=\frac{Q_{1} a_{1}-Q_{1} a_{1}^{\min }}{Q_{1} a_{1}^{\max }-Q_{1} a_{1}^{\min }}, Q_{2}=\frac{Q_{2}-Q_{2}^{\min }}{Q_{2}^{\max }-Q_{2}^{\min }},
$$




$$
\Delta K=\frac{\Delta K-\Delta K^{\min }}{\Delta K^{\max }-\Delta K^{\min }}
$$

where $Q_{1}$ and $a_{1}$ are the heat flux and the crack length in the first stage, $Q_{2}$ and $a_{2}$ are the heat flux and the crack length in the second stage. The superscripts "max" and "min" denote maximum and minimum values during the fatigue test.

\section{CONCLUSION}

A series of experiments was carried out to study the energy dissipation at the fatigue crack tip under biaxial loading. The experimental technique for measuring the energy dissipation during fatigue test under biaxial loading was developed based on the contact heat flux sensor and the method of infrared thermography. The results of this study confirm the hypothesis for the existence of two modes of energy dissipation during the fatigue cracks propagation. The first mode corresponds to slowly propagating cracks $(10 \mathrm{e}-7-10 \mathrm{e}-5 \mathrm{~m} / \mathrm{cycle})$ and is described by the relation $\left(\frac{d a}{d N} \sim Q_{1} a_{1}\right)$.The point corresponding to a change in the character of energy dissipation lies on the linear section of the Paris curve. The obtained experimental results allows us to generalize the hypothesis about the linear relationship between the energy dissipation at the fatigue crack tip and its growth rate to the case of biaxial loading. The study revealed qualitative agreement between the energy approach to the description of the fatigue crack propagation and the classical approach, which is based on the use of the stress intensity factor.

\section{ACKNOWLEDGMENTS}

7 his work was supported by the grant of the President of Russian Federation for support of young Russian scientists and leading scientific schools [MK-1236.2017.1] and the Russian Foundation for Basic research [grant number 16-48-590148].

\section{REFERENCES}

[1] Yates, J.R., Zanganeh, M., Tomlinson, R.A., Brown, M.W., DiazGarrido F.A. (2008). Crack paths under mixed mode loading, Engineering Fracture Mechanics, 75(3-4), pp. 319-330.

[2] Mokhtarishirazabad, M., Lopez-Crespo, P., Moreno, B., Lopez-Moreno, A., Zanganeh M. (2017). Optical and analytical investigation of overloads in biaxial fatigue cracks, International Journal of Fatigue, 100(2),pp. 583-590.

[3] Izumi, Y., Sakagami, T., Yasumura, K., Shiozawa, D. (2014). A new approach for evaluating stress intensity factor based on thermoelastic stress analysis, APCFS/SIF, pp. 47-51.

[4] Short, J. S., Hoeppner, D. W. (1989). A Global/local theory of fatigue crack propagation, Engineering Fracture mechanics, 33(2), pp. 175-184.

[5] Meneghetti, G., Ricotta, M. (2016). Evaluating the heat energy dissipated in a small volume surrounding the tip of a fatigue crack, International Journal of Fatigue, 92(2), pp. 605-615.

[6] Risitano, A., Risitano, G. (2013). Cumulative damage evaluation in multiple cycle fatigue tests taking into account energy parameters, International Journal of Fatigue, 48, pp. 214-222.

[7] Pradere, C., Joanicot, M., Batsale, J-C., Toutain, J., Gourdon, C. (2006). Processing of temperature field in chemical microreactors with infrared thermography, QIRT Journal, 3, pp. 117-135.

[8] Matvienko, Yu.G., Morozov, E M. (2004). Calculation of the energy J-integral for bodies with notches and cracks, International Journal of Fracture, 125, pp. 249-261.

[9] Rosakis, P., Rosakis, A.J., Ravichandran, G., Hodowany, J. (2000). A thermodynamic internal variable model for the partitional of plastic work into heat and stored energy in metals, J. Mech. Phys. Solids, 48, pp. 581-607.

[10] Oliferuk, W., Maj, M., Raniecki, B. (2004). Experimental Analysis of Energy Storage Rate Components during Tensile Deformation of Polycrystals, Materials Science and Engineering, 374, pp. 77-81.

[11] Izyumova, A., Plekhov, O. (2014). Calculation of the energy J-integral in plastic zone ahead of a crack tip by infrared scanning, FFEMS, 37, pp. 1330-1337. 
[12] Farren, W.S., Taylor, G.I. (1925). The heat developed during plastic extension of metals, Proc. Royal. Soc. of London. Ser., 107, pp. 422-451.

[13] Vshivkov, A., Iziumova, A., Bar, U., Plekhov, O. (2016). Experimental study of heat dissipation at the crack tip during fatigue crack propagation, Fracture and Structural Integrity, 35, pp. 57-63. 